\title{
Optimal investment decisions with a liability: The case of defined benefit pension plans
}

\author{
Ricardo Josa-Fombellida ${ }^{\mathrm{a}}$, Juan Pablo Rincón-Zapatero ${ }^{\mathrm{b}, *}$ \\ a Departamento de Estadística e Investigación Operativa, Universidad de Valladolid, Paseo Prado de la Magdalena, s/n 47005 Valladolid, Spain \\ bepartamento de Economía, Universidad Carlos III de Madrid, C/ Madrid, 126-128, 28903 Getafe, Madrid, Spain
}

In this paper the optimal management of an aggregated dynamic pension fund is studied. To cover the promised liabilities to workers at the age of retirement, the plan sponsor continuously manages time-varying funds. He or she can choose the rate of contribution to the fund, the investment in a given number of risky assets, and a security with constant rate of return. The problem of maximizing the probability that the fund assets achieve some prescribed goal before some undesirable lower value, or ruin point, is first considered. Secondly, the problem of minimizing (resp. maximizing) the expected discounted cost of reaching a ruin point (resp. beating a desired objective) is solved. Finally, maximization of utility function when the fund can suddenly terminate is analyzed. The main finding is that optimal investment policies are of constant proportionality type.

JEL classification: G23; G11

Subject and Insurance Branch Codes: E13; B81

Keywords: Pension funding; Risk management; Stochastic control; Portfolio theory

\section{Introduction}

Pension funds currently represent one of the most important institutions in financial markets, because of their high investment capacity and because they complement the role of the government, allowing those workers that have reached retirement age to maintain their standard of living. These two aspects justify the interest generated over recent years in the study of the optimum management of pension plans.

There are two principal alternatives in the design of pension plans in correspondence to the assignation of risk. In a defined contribution plan, the risk derived from the fund management is borne by the beneficiary. However, in a defined benefit plan, where the benefits are normally related to the final salary level, the financial risk is assumed by the sponsor agent.

\footnotetext{
* Corresponding author. Tel.: +349162486 66; fax: +34916249875.

E-mail addresses: ricar@eio.uva.es (R. Josa-Fombellida), jrincon@eco.uc3m.es (J.P. Rincón-Zapatero).
} 
This paper studies the model of a defined benefit plan, as described in Haberman and Sung (1994), Boulier et al. (1995), Cairns (2000) or Josa-Fombellida and Rincón-Zapatero (2001, 2004). In all these papers the objective of the sponsor is to keep the fund assets as close as possible to the actuarial liability. To this end, the plan sponsor can choose the rate of contribution to the fund, the investment in a given number of risky assets, and a security with constant rate of return. The optimal contribution rate corresponds to what, in the literature, is called a spread method of funding. This type of funding method is quite popular in actuarial practice, because of its simplicity and good stability properties. Bowers et al. (1979), Cairns and Parker (1997) and Haberman et al. (2000) constitute examples of the use of this method in the literature.

In this paper we consider the problem from a different point of view. We look at three different objectives for the sponsor, which at first sight seem to be different and mutually exclusive, but in fact it turns out that, with adequate selection of the parameters involved, some of them are attained simultaneously. A summary of the cases where this happens is given in Tables 5 and 6 at the end of the paper. We consider the following cases. First, the sponsor wishes to maximize the probability that the fund assets achieve a fixed target value, before a fixed bankruptcy value. Second, the sponsor's aim is to minimize the expected discounted penalization cost associated with an inadmissibly high value of the unfunded actuarial liability, or to maximize the expected discounted reward of attaining a given surplus level. These types of problem, which mathematically are of stochastic control of Dirichlet type, have been considered in Pestien and Sudderth (1985) and more recently in Browne $(1997,1999)$ in different contexts. In the third and last case, the sponsor maximizes some utility function along an infinite horizon, when the date of abrupt termination of the funding process is random, with exponential distribution.

The main result is that, in all cases, the optimal investment decisions are proportional to the unfunded actuarial liability, which is the difference between the liabilities and the fund assets. Hence the optimal policies require more risk to be assumed when this variable takes a larger value than when it is near zero.

The paper is organized as follows. Section 2 defines the elements of the pension scheme and describes the financial market where the fund is investing. Under the assumption that the contributions are of spread method type, the evolution of the fund assets is obtained. In Section 3 we solve the problem of maximizing the probability that the unfunded actuarial liability decreases to zero before it reaches a ruin value. In Section 4 the problem of minimizing the cost (resp. maximizing a recompense) of reaching a ruin point (resp. of attaining a target fund level) is analyzed. Section 5 shows the optimal investment when there is a positive probability of sudden termination of the pension plan. Finally, Section 6 is dedicated to establishing some conclusions. In Appendix A the general results that we need on stochastic control are stated. All proofs are in Appendix B.

\section{The pension model}

The pension plan that we take into account is of aggregated type, thus the variables listed below refer to the total group of participants. To cover the promised liabilities to the workers at the age of retirement, the plan's sponsor continuously withdraws time-varying funds. The principal elements intervening in the funding process and the essential hypotheses allowing its temporary evolution to be determined are as follows.

$F(t) \quad$ Value of fund assets at time $t$.

$P(t) \quad$ Benefits promised to the participants at time $t$, which we suppose to be deterministic. These are related to the salary at the moment of retirement.

$C(t) \quad$ Contribution rate made by the sponsor to the funding process at time $t$.

$A L(t) \quad$ Actuarial liability at time $t$, that is, total liabilities of the sponsor.

$N C(t) \quad$ Normal cost at time $t$; if the fund assets match the actuarial liability, and if there are no uncertain elements in the plan, the normal cost is the value of the contributions allowing equality between asset funds and obligations.

$S C(t) \quad$ Supplementary cost at time $t$, equal to $C(t)-N C(t)$.

$M(s) \quad$ Percentage of the value of the future benefits accumulated until age $s \in[a, d]$, where $a$ is the common age of entrance into the fund and $d$ is the common age of retirement.

$\delta \quad$ Constant rate of valuation of the liabilities, which can be specified by the regulatory authorities.

We suppose that functions $P$ and $M$ are both differentiable. 
As mentioned in the Introduction, a spread method of fund amortization will be used. Thus we will assume that the supplementary contribution rate is proportional to the unfunded actuarial liability, $S C(t)=k(A L(t)-F(t))$, that is

$$
C(t)=N C(t)+k(A L(t)-F(t)),
$$

with $k$ being a constant selected by the employer, representing the rate at which surplus or deficit is amortized. A negative value of $k$ when the fund is underfunded (resp. overfunded) means that the contribution is below (resp. above) the normal cost. Actuarial practice takes $1 / k$ to be equal to a continuous annuity with amortization over $m$ years, an extreme case being a perpetual annuity, $m=\infty$. However, some theoretical studies such as O'Brien (1987), Haberman and Sung (1994) or Josa-Fombellida and Rincón-Zapatero $(2001,2004)$, in a framework close to this paper, show that proportional funding, $S C=k(A L-F)$, is optimal with $1 / k$ not necessarily given by an annuity. Instead, parameters defining the preferences of the sponsor and some characteristics of the stock market enter into the definition of $k$; we refer the reader to the aforementioned papers for the exact details. Hence, we consider an alternative range for $k$ to what actuarial practice suggests. Furthermore, the control problems that we study are degenerated for some values of $k$, in the sense that they are not truly stochastic problems or that they do not have a solution, at least such as these problems are settled here - without constraints affecting investment decisions.

The actuarial functions $A L$ and $N C$ are

$$
\begin{aligned}
& A L(t)=\int_{a}^{d} \mathrm{e}^{-\delta(d-s)} P(t+d-s) M(s) \mathrm{d} s, \\
& N C(t)=\int_{a}^{d} \mathrm{e}^{-\delta(d-s)} P(t+d-s) M^{\prime}(s) \mathrm{d} s,
\end{aligned}
$$

respectively, and they are linked by the ordinary differential equation

$$
A L^{\prime}(t)=\delta A L(t)+N C(t)-P(t), \quad t \geq 0,
$$

as proven in Bowers et al. (1979).

The function $M$ satisfies $0 \leq M(s) \leq 1$ for all $s, M(s)=0$ for $s \leq a$, and $M(s)=1$ for $s \geq d$. The simplest case is when the number of employees accumulates uniformly along the interval $[a, d]$, that is, $M(s)=(s-a)(d-a)^{-1}, a<s<d$. Aside from the constant benefit case, a usual model is the exponential model, where it is supposed that the benefits, as well the salary, grow at an exponential rate. In this case, $A L$ and $N C$ are also exponential functions. We do not restrict ourselves to this model.

In the rest of this section we describe the financial market where the fund operates. Given an $n$-dimensional standard Brownian motion $w=\left(w_{1}, \ldots, w_{n}\right)^{\top}$, we consider the complete probability space $(\Omega, \mathcal{F}, \mathbb{P})$ generated by it, that is to say, $\mathcal{F}$ is the filtration $\left\{\mathcal{F}_{t}\right\}_{t \geq 0}$, with $\mathcal{F}_{t}=\sigma\left\{w_{1}(s), \ldots, w_{n}(s) ; 0 \leq s \leq t\right\}$.

The plan sponsor manages the fund by means of a portfolio formed by $n$ risky assets $\left\{S^{i}\right\}_{i=1}^{n}$, which are correlated geometric Brownian motions generated by $w$, and a riskless asset $S^{0}$, as proposed in Merton (1971), that is, whose evolutions are given by the equations:

$$
\begin{aligned}
& \mathrm{d} S^{0}(t)=r S^{0}(t) \mathrm{d} t, \quad \text { with } r>0, \\
& \mathrm{~d} S^{i}(t)=S^{i}(t)\left(b_{i} \mathrm{~d} t+\sum_{j=1}^{n} \sigma_{i j} \mathrm{~d} w_{j}(t)\right), \quad i=1,2, \ldots, n .
\end{aligned}
$$

It is assumed that $b_{i}>r$ for all $i$, so the sponsor has incentives to invest with risk. The amount of fund invested in time $t$ in the risky asset $S^{i}$ is denoted by $\lambda_{i}(t), i=1,2, \ldots, n$. The remainder, $F(t)-\sum_{i=1}^{n} \lambda_{i}(t)$, is invested in the bond. Borrowing and shortselling is allowed. A negative value of $\lambda_{i}$ means that the sponsor sells a part of his risky asset $S^{i}$ short while, if $\sum_{i=1}^{n} \lambda_{i}$ is larger than $F$, then he or she gets into debt to purchase the stocks, borrowing at the riskless interest rate $r$. In all cases studied, the efficient portfolio does not imply short-selling. We suppose that $\{\boldsymbol{\Lambda}(t): t \geq 0\}$, with $\boldsymbol{\Lambda}(t)=\left(\lambda_{1}(t), \lambda_{2}(t), \ldots, \lambda_{n}(t)\right)^{\top}$, is a control process adapted to filtration $\left\{\mathcal{F}_{t}\right\}_{t \geq 0}, \mathcal{F}_{t}$-measurable, Markovian and stationary, satisfying $\mathbb{E} \int_{0}^{s} \boldsymbol{\Lambda}(t)^{\top} \boldsymbol{\Lambda}(t) \mathrm{d} t<\infty$, for all $s$. 
Therefore, the fund dynamic evolution under the investment policy $\boldsymbol{\Lambda}$ is:

$$
\mathrm{d} F(t)=\sum_{i=1}^{n} \lambda_{i}(t) \frac{\mathrm{d} S^{i}(t)}{S^{i}(t)}+\left(F(t)-\sum_{i=1}^{n} \lambda_{i}(t)\right) \frac{\mathrm{d} S^{0}(t)}{S^{0}(t)}+(C(t)-P(t)) \mathrm{d} t .
$$

By substituting (3) and (4) in (5), we obtain:

$$
\mathrm{d} F(t)=\left(r F(t)+\sum_{i=1}^{n} \lambda_{i}(t)\left(b_{i}-r\right)+C(t)-P(t)\right) \mathrm{d} t+\sum_{i=1}^{n} \sum_{j=1}^{n} \lambda_{i}(t) \sigma_{i j} \mathrm{~d} w_{j}(t),
$$

with initial condition $F(0)>0$.

Next, we will assume the notation $\sigma=\left(\sigma_{i j}\right), \mathbf{b}=\left(b_{1}, b_{2}, \ldots, b_{n}\right)^{\top}, \mathbf{1}=(1,1, \ldots, 1)^{\top}$, and $\Sigma=\sigma \sigma^{\top}$. We take as given the existence of $\Sigma^{-1}$, that is to say, $\sigma^{-1}$. Finally, the vector of standardized risk premia or Sharpe ratio of the portfolio is denoted by $\theta=\sigma^{-1}(\mathbf{b}-r \mathbf{1})$.

So, we can write (6) as:

$$
\mathrm{d} F(t)=\left(r F(t)+\boldsymbol{\Lambda}^{\top}(t)(\mathbf{b}-r \mathbf{1})+C(t)-P(t)\right) \mathrm{d} t+\boldsymbol{\Lambda}^{\top}(t) \sigma \mathrm{d} w(t),
$$

which, with the initial condition $F(0)$, determines the fund evolution. Substituting (1) into Eq. (7), it takes the form

$$
\mathrm{d} F(t)=\left(r F(t)+\boldsymbol{\Lambda}^{\top}(t)(\mathbf{b}-r \mathbf{1})+N C(t)+k(A L(t)-F(t))-P(t)\right) \mathrm{d} t+\boldsymbol{\Lambda}^{\top}(t) \sigma \mathrm{d} w(t) .
$$

We assume throughout the paper that the technical interest rate coincides with the rate of return of the bond, that is, $\delta=r$, although the results could be extended to $\delta \neq r$.

So, by (2), in terms of $X=F-A L$, Eq. (8) reads:

$$
\mathrm{d} X(t)=\left((r-k) X(t)+\boldsymbol{\Lambda}^{\top}(t)(\mathbf{b}-r \mathbf{1})\right) \mathrm{d} t+\boldsymbol{\Lambda}^{\top}(t) \sigma \mathrm{d} w(t),
$$

with the initial condition $X(0)=x$. Note that, when $X<0, k>0$ has the effect of diminishing (augmenting when $k<0$ ) the rate of interest that is being charged on the unfunded liability. The situation is reversed if $X>0$.

To stress the influence of the investment vector, we will write $X^{\Lambda}(t)$ instead of $X(t)$. Furthermore, since we are going to use dynamic programming, we need to solve the optimization problem for every initial condition $X(0)$, which we denote by $x$. Hence $X^{\boldsymbol{\Lambda}}(t)=F(t)-A L(t)$ as a process, but $x=F-A L$ as a fixed initial value.

It is important to emphasize that, although the functional form of the contributions is fixed in (1), its evolution is uncertain, depending on the fund assets. In the following, we consider the new variable $X=F-A L$.

\section{Maximizing the probability of reaching an objective}

In this section we analyze the case where the objective of the sponsor is to maximize the probability that $X$ reaches the value 0 prior to another undesirable value, denoted $\ell=\bar{F}-A L$. The value of $\bar{F}<A L$ could be imposed by the authorities as a legal minimum floor below which fund assets cannot go, because it is considered as a non-return point or "ruin" point. Unfortunately, the problem formulated like so does not have a solution, as will be shown in Remark 3.3. As a consequence, we set the problem as follows: maximize the probability that $X$ reaches the value $u$ before the ruin value $\ell$, with $\ell<u<0$ and $u$ as close to 0 as desired. Note that here we are considering the case where the fund is below $A L$ (underfunded case). In the region where the fund is over $A L$ (overfunded case), the objective is to increase $X$ as much as possible, so the problem is to maximize the probability that $X$ reaches $u$ before $\ell$ with $0<\ell<u$. The formulations of the problem in both regions are quite similar, but the hypotheses needed and the results obtained are different.

Let us denote by $U(x)$ the maximum value of the probability to attain $u$ prior to $\ell$ when $\ell<x<u$ :

$$
U(x)=\sup _{\boldsymbol{\Lambda} \in \mathcal{A}_{x}} \mathbb{P}_{x}\left(\tau_{\ell}^{\boldsymbol{\Lambda}}>\tau_{u}^{\boldsymbol{\Lambda}}\right)=\sup _{\boldsymbol{\Lambda} \in \mathcal{A}_{x}} \mathbb{P}_{x}\left(\tau_{\ell u}^{\boldsymbol{\Lambda}}=\tau_{u}^{\boldsymbol{\Lambda}}\right),
$$

where, for instance, $\tau_{\ell}^{\boldsymbol{\Lambda}}$ is the first time $X$ hits the value $\ell$ when the investment policy is $\boldsymbol{\Lambda}$. See Appendix A for the definitions and notation. 


\subsection{Underfunded region, $u<0$}

In this case we restrict our attention to $k<r$. When the contribution effort is less than the force of interest, fund dynamics might cease to be mean reverting because the amortization rate is not strong enough to overcome the effect of interest on the fund, so there is a positive probability of ruin. In contrast, as shown by (9), when $k \geq r$ the problem is not a genuine ruin problem, since that full investment in the bond ensures that $X$ is strictly increasing $(k>r)$ or constant $(k=r)$. However, when $k<r, 100 \%$ investment in cash leads the fund to the ruin point. In the real world, pension managers choose $k \geq r$, with equality if $1 / k$ is the value of a perpetual annuity at interest rate $r$. As we have just seen, this practice avoids ruin. However, it is interesting to elucidate if there is or is not a mechanism minimizing the probability of ruin, even if the contribution rate effort $k$ is less than $r$, because total expected contribution could then be lowered. The next result shows that such a mechanism exists and is, of course, a sensible portfolio management. Here is a typical tradeoff problem in pension fund management: lowering contributions at the expense of taking on more risk.

In the next proposition, recall that the market price of risk $\theta$ is defined as $\theta=\sigma^{-1}(\mathbf{b}-r \mathbf{1})$.

Proposition 3.1. Assume that $k<r$ and $\ell<x<u<0$. The optimal investment strategy is given by

$$
\boldsymbol{\Lambda}_{U}(X)=-\frac{2(r-k)}{\theta^{\top} \theta} \Sigma^{-1}(\mathbf{b}-r \mathbf{1}) X=\frac{2(r-k)}{\theta^{\top} \theta} \Sigma^{-1}(\mathbf{b}-r \mathbf{1})(A L-F)
$$

and the value function is given by

$$
U(x)=\frac{|x|^{\alpha}-|\ell|^{\alpha}}{|u|^{\alpha}-|\ell|^{\alpha}}=\frac{(A L-F)^{\alpha}-|\ell|^{\alpha}}{|u|^{\alpha}-|\ell|^{\alpha}},
$$

where $\alpha=1+\theta^{\top} \theta /(2(r-k))$.

The policy $\boldsymbol{\Lambda}_{U}$ given by (10) is of constant proportion type. These policies are widely used in practice, due to their simplicity and because they appear as optimal investment strategies in many related problems (see e.g. Pestien and Sudderth, 1985; Browne, 1998). The optimal behavior of the sponsor is to allocate fixed constant proportions of the difference of the obligations and the fund assets, taking on more risk when the difference is large. In fact, the risky investment is a maximum near the ruin point, when $F=\bar{F}$, and then it decreases as the fund gets closer to $A L$. Since the optimal solution does not depend on $\ell$ and $u$, it is clear that this policy also minimizes the probability of ruin.

The role of $k$ in the solution agrees with commonsense: an increase in $k$, that is, an increase in the contributions, allows a reduction in the amount invested in the portfolio. Then, if the plan's sponsor or the employees wish to reduce the contributions, it must be compensated for by taking a greater risk.

Remark 3.1. Although investment strategies do not involve shortselling, borrowing can be necessary. The sponsor borrows money at rate $r$ to invest in asset $i, \lambda_{i, U} \geq F$, if and only if $0 \leq F \leq v_{i} A L$, with $v_{i}=\mathbf{e}_{i} \Sigma^{-1}(\mathbf{b}-r \mathbf{1}) /(\alpha-$ $\left.1+\mathbf{e}_{i} \Sigma^{-1}(\mathbf{b}-r \mathbf{1})\right)$, where $\mathbf{e}_{i}$ is defined as $\mathbf{e}_{i}=(0, \ldots, \stackrel{(i)}{1}, \ldots, 0)$. He or she must borrow to invest in some stock, $\sum_{i=1}^{n} \lambda_{i, U} \geq F$, if and only if $0 \leq F \leq \nu_{0} A L$, with $\nu_{0}=\mathbf{1}^{\top} \Sigma^{-1}(\mathbf{b}-r \mathbf{1}) /\left(\alpha-1+\mathbf{1}^{\top} \Sigma^{-1}(\mathbf{b}-r \mathbf{1})\right)$.

Remark 3.2. Under policy $\boldsymbol{\Lambda}_{U}$, (9) is

$$
\mathrm{d} X^{\Lambda_{U}}(t)=-(r-k) X^{\Lambda_{U}}(t) \mathrm{d} t-\frac{2(r-k)}{\theta^{\top} \theta} X^{\Lambda_{U}}(t) \theta^{\top} \mathrm{d} w(t), \quad \text { for } 0 \leq t \leq \tau_{\ell u}^{\boldsymbol{\Lambda}},
$$

which is a geometric Brownian motion with drift parameter $-(r-k) X$ and diffusion coefficient (at the square) $4(r-k)^{2} X^{2}$. Therefore, it is immediate to check that

$$
\mathbb{P}_{x}\left(\tau_{\ell}>\tau_{u}\right)=\frac{\psi(x)-\psi(\ell)}{\psi(u)-\psi(\ell)}=U(x),
$$

where $\psi$ is the associated scale function to the stochastic process $X^{\boldsymbol{\Lambda}_{U}}$; see Karlin and Taylor (1981).

Remark 3.3. We have found an investment policy maximizing the probability of reaching $u$ before $\ell<u$ for every $u<0$. It is easy to see that it is impossible to achieve $u=0$ with this policy in finite time. This is 
because 0 is an unattainable barrier for a geometric Brownian motion, as shown in Karlin and Taylor (1981). Thus $\tau_{0}^{\boldsymbol{\Lambda}_{U}}=\infty$ and there is no optimal policy for the problem. Following Browne (1997), $\varepsilon$-optimal strategies could be constructed.

Illustration of the results. It is worth comparing the total size of expected contributions when $k<r$ and $k^{\prime}>r$, respectively. Recall that, in the former case, the optimal strategy is given by (10), whereas for the latter, full investment in the bond is assumed. Our aim is to show that active management of the fund can diminish the total expected discounted contributions, at the expense of incurring some small positive probability of ruin. After some theoretical results are provided - the analytical expression for the expected time of exit of the interval $(\ell, u)$, and for the expected value of contributions - we proceed to carry out some numerical illustrations of that fact. As the stochastic problem is solved in closed form, the sponsor can decide ex ante which value of $k$ to choose, since she/he knows exactly the dependence of the outcome with respect to $k$. For a given environment, the selection of $k$ fully determines the probability of success and the size of total expected contributions. It is a discretional decision of the sponsor to choose the more satisfactory combination of admissible shortfall probability/amortization effort possibilities. We will distinguish two different attitudes of the sponsor: one is named sensible management and corresponds to a selection of $k<r$, indicating that the sponsor is willing to incur some risk if it is possible to considerably reduce total expected amortization; the other is designed as secure management, $k^{\prime}>r$ and $\boldsymbol{\Lambda} \equiv 0$, for a sponsor that prefers driving the fund to a higher value for sure, though at the expense of higher amortization effort and longer periods of time until reaching the objective. The reader must be aware that the property does not hold for every admissible value of the parameters defining our problem, but for a relevant range of values that we think is sound in application. Because of the variety in the intervening parameters, it seems that is is not easy to prove the general properties of the fund attaining the size of expected total contributions under sensible/secure management. For this reason, we concentrate on some numerical experiments.

\section{Expected value of total contribution with sensible management, $k<r$}

Let us consider $T(x)=\mathbb{E}_{x} \tau_{\ell u}^{\Lambda}$ and $\mathbb{E}_{x} \int_{0}^{\tau_{\ell u}^{\Lambda}} \mathrm{e}^{-r t} C^{k}(t) \mathrm{d} t$, the expected time of exit of the interval $(\ell, u)$ of $X^{\boldsymbol{\Lambda}_{U}}$, and the accumulated expected value of discounted contributions until exit of the interval $(\ell, u)$, respectively, with $\ell<x=X^{\boldsymbol{\Lambda}_{U}}(0)<u<0$. By the definition in (1),

$$
\mathbb{E}_{x} \int_{0}^{\tau_{\ell u}^{\Lambda}} \mathrm{e}^{-r t} C^{k}(t) \mathrm{d} t=\mathbb{E}_{x} \int_{0}^{\tau_{\ell u}^{\Lambda}} \mathrm{e}^{-r t} N C(t) \mathrm{d} t-k \mathbb{E}_{x} \int_{0}^{\tau_{\ell u}^{\Lambda}} \mathrm{e}^{-r t} X^{\Lambda_{U}}(t) \mathrm{d} t \equiv \frac{N C}{r}(1-R(x))-k S(x),
$$

with $R(x)$ denoting $\mathbb{E}_{x}\left(\mathrm{e}^{-r \tau_{\ell u}^{\Lambda}}\right)$ and where we have supposed that benefits, $P$, are constant. As a consequence, $A L$ and $N C$ are also constant. Exact expressions could also be obtained for the exponential fund model at little cost at the expense of introducing a new parameter. We restrict ourselves for the remainder of this section to the constant case, in order to highlight the main features of the model. Following Karlin and Taylor (1981, pp. 192-204) or Harrison (1985), it is possible to characterize the functions $R, S$ and $T$ as solutions of some second order differential equations when $X^{\boldsymbol{\Lambda}_{U}}$ is a general diffusion process. In our model we deal with a geometric Brownian motion (see Remark 3.2), allowing us to find the functions in closed form, because those differential equations are easily transformed into linear equations. We do not go further into the details, because they are quite standard. ${ }^{1}$

$$
\begin{aligned}
& R(x)=\frac{1}{\Delta}\left(\left(|u|^{m_{2}}-|\ell|^{m_{2}}\right)|x|^{m_{1}}+\left(|\ell|^{m_{1}}-|u|^{m_{1}}\right)|x|^{m_{2}}\right), \\
& S(x)=\frac{1}{2 r-k}\left(|x|+\frac{1}{\Delta}\left(\left(|u||\ell|^{m_{2}}-|\ell||u|^{m_{2}}\right)|x|^{m_{1}}+\left(|\ell||u|^{m_{1}}-|u||\ell|^{m_{1}}\right)|x|^{m_{2}}\right)\right), \\
& T(x)=\frac{\alpha-1}{(r-k) \alpha}\left(\ln \left(\frac{x}{\ell}\right)-U(x) \ln \left(\frac{u}{\ell}\right)\right),
\end{aligned}
$$

\footnotetext{
${ }^{1} R$ satisfies $\frac{2(r-k)^{2}}{\theta^{\top} \theta} x^{2} R^{\prime \prime}-(r-k) x R^{\prime}-r R=0$, with $R(\ell)=R(u)=1, S$ satisfies $\frac{2(r-k)^{2}}{\theta^{\top} \theta} x^{2} S^{\prime \prime}-(r-k) x S^{\prime}-r S+x=0$, with $S(\ell)=S(u)=0$, and $T$ satisfies $\frac{2(r-k)^{2}}{\theta^{\top} \theta} x^{2} T^{\prime \prime}-(r-k) x T^{\prime}+1=0$, with $T(\ell)=T(u)=0$. It is well known that these Euler-type equations are linearized by the change variable $x=-\mathrm{e}^{t}$.
} 
with $m_{1,2}$ the roots (they are always real in our framework) of

$$
\frac{2(r-k)^{2}}{\theta^{\top} \theta} y^{2}-\left((r-k)+\frac{2(r-k)^{2}}{\theta^{\top} \theta}\right) y-r=0 .
$$

In the above we are assuming that $m_{1,2} \neq 1$ and that $\Delta=|\ell|^{m_{1}}|u|^{m_{2}}-|\ell|^{m_{2}}|u|^{m_{1}}$ does not vanish, in order to shorten the cases under study. Note that we have written the corresponding expressions in terms of the function $U$ and of the parameter $\alpha$ given in Proposition 3.1. Of course, we are considering $k<r$.

Total value of contribution with secure management, $k^{\prime}>r$ and $\mathbf{\Lambda}=0$

We denote $k$ with $k^{\prime}$ to distinguish from the above case. The time of hitting $u$ from $x$ for the process $X^{0}$ is $\bar{t}(x)=\ln (u / x) /\left(r-k^{\prime}\right)$ and the accumulated amount of $X^{0}$ up to time $\bar{t}(x)$ is $\bar{x}=(u-x) /\left(r-k^{\prime}\right)$. Denoting $C^{k^{\prime}}$ as the total discounted contribution in this case and taking into account that we suppose $N C$ to be constant, then

$$
C^{k^{\prime}}(x)=\frac{N C}{r}\left(1-\left(\frac{u}{x}\right)^{\frac{r}{k^{\prime}-r}}\right)-x\left(1-\left(\frac{u}{x}\right)^{\frac{k^{\prime}}{k^{\prime}-r}}\right) .
$$

We suppose that $\ell, u$ and $x$ are proportional to $A L$, with coefficients between -1 and 0 , which will be specified next.

We intend to address the following question: with the parameters chosen, is it possible to use a sensible management of the fund by selecting a risky portfolio, such that the total expected discounted contribution is lesser than that with secure management, and at the same time reducing ruin probabilities to a comfortable level for the sponsor? To answer this question partially, some numerical results are shown for selected values of the parameters.

Assumptions. The assumptions that we consider are the following:

- $P=10$ is constant, ${ }^{2}$ the entry age to the fund is $a=25$ years, the age of retirement is $d=65$ years, and the accrual function is $M(s)=(d-s) /(d-a) ; M(s)=(s-a) /(d-a)$.

- The risk-free rate of interest is $r=5 \%$.

- Risky investment is only in one asset; we consider three cases depending on the Sharpe ratio: $\theta=0.25,0.30$ and 0.35 . Typical parameters leading to these values are $b=10 \%$, and $\sigma=1 / 5, \sigma=1 / 6$, and $\sigma=1 / 7$, respectively.

- $k^{\prime}$ is the inverse of an annuity of $m=20$ years, calculated at the rate $i=\mathrm{e}^{r}-1, k^{\prime} \approx 8.11 \%$; hence we choose an intermediate value for the amortization period $m$, lesser than the future working lifetime of the members, indicating that the sponsor is concerned with a moderately rapid elimination of deficit.

- $\bar{F}=0.5 A L$ is considered to be the ruin point by the sponsor, hence $\ell=-0.5 A L$, and the initial value of the fund is $F(0)=0.8 A L$, or $x=-0.2 A L$.

- Three different values are considered for the target $u: F=0.81 A L(u=-0.19 A L), F=0.82 A L(u=-0.18 A L)$ and $F=0.84 A L(u=-0.16 A L)$; that is, the sponsor wishes to maximize the probability of debt reduction by percentages $5 \%, 10 \%$ and $20 \%$, respectively.

Results. ${ }^{3}$ It is important to highlight that we are supposing that the ruin event is not $F=0$ but when the fund wealth is one half the actuarial liability. So, we must be cautionary about the meaning of "ruin point" in this framework. It can suggest a very dramatic situation for the fund, whereas actually the sponsor still has a margin of safety in the management of fund assets.

The numerical experiments allow us to conclude that the size of $\theta$ has a major impact on both the probability and the expected time of reaching the objectives, and on the total value of discounted expected contributions. Table 1 shows the dependence with respect to the Sharpe ratio of the minimum probability of ruin, supporting the same total expected contribution in both types of management. Of course, the sponsor prefers secure management in this case,

\footnotetext{
${ }^{2}$ This implies, join with the following assumption that $N C=4.3233$ and $A L=113.5335$. There is not a significative change in the results obtained by selecting other values for $P$.

3 The following calculations are merely illustrative, as the figures that appear have been truncated to the fourth or the second decimal place. For the computations, some symbolic and numerical features of DERIVE have been used.
} 
Table 1

Impact of $\theta$ on the minimum probability of ruin, equating expected contributions in both types of management ${ }^{\mathrm{a}}$

\begin{tabular}{|c|c|c|c|c|}
\hline \multirow[t]{2}{*}{ Sharpe ratio } & \multicolumn{4}{|c|}{ Probability of ruin, $\%$} \\
\hline & \multicolumn{4}{|c|}{ Debt reduction } \\
\hline 0.25 & 1.11 & 2.11 & 3.80 & 5.16 \\
\hline 0.30 & 0.85 & 1.61 & 2.86 & 3.85 \\
\hline 0.35 & 0.64 & 1.20 & 2.11 & 2.80 \\
\hline
\end{tabular}

a Parameter values: $\ell=-0.5 A L, x=-0.2 A L, r=5 \%, P=10$.

Table 2

Performance of sensible management with respect to secure management with debt reduction of $5 \%(\bar{t}=1.65)^{\mathrm{b}}$

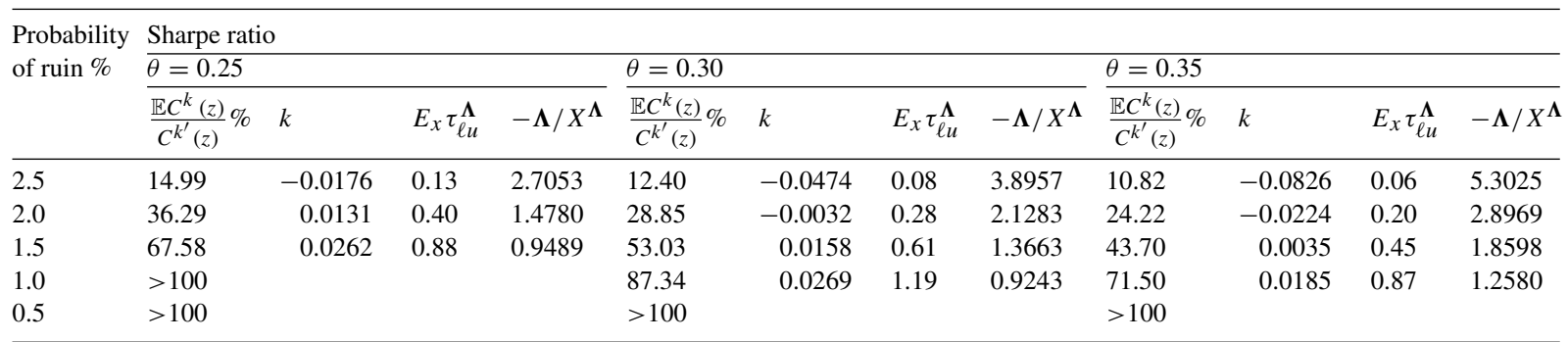

b Parameter values: $\ell=-0.5 A L, x=-0.2 A L, u=-0.19 A L, \theta=0.25(\sigma=1 / 5), \theta=0.3(\sigma=1 / 6), \theta=0.35(\sigma=1 / 7), r=5 \%, P=10$.

Table 3

Performance of sensible management with respect to secure management with debt reduction of $10 \%(\bar{t}=3.39)^{\mathrm{c}}$

\begin{tabular}{|c|c|c|c|c|c|c|c|c|c|c|c|c|}
\hline \multirow{3}{*}{$\begin{array}{l}\text { Probability } \\
\text { of ruin } \%\end{array}$} & \multicolumn{12}{|c|}{ Sharpe ratio } \\
\hline & \multicolumn{4}{|c|}{$\theta=0.25$} & \multicolumn{4}{|l|}{$\theta=0.30$} & \multicolumn{4}{|l|}{$\theta=0.35$} \\
\hline & $\frac{\mathbb{E} C^{k}(z)}{C^{k^{\prime}}(z)} \%$ & $k$ & $E_{x} \tau_{\ell u}^{\boldsymbol{\Lambda}}$ & $-\boldsymbol{\Lambda} / X^{\boldsymbol{\Lambda}}$ & $\frac{\mathbb{E} C^{k}(z)}{C^{k^{\prime}}(z)} \%$ & $k$ & $E_{x} \tau_{\ell u}^{\boldsymbol{\Lambda}}$ & $-\boldsymbol{\Lambda} / X^{\boldsymbol{\Lambda}}$ & $\frac{\mathbb{E} C^{k}(z)}{C^{k^{\prime}}(z)} \%$ & $k$ & $E_{x} \tau_{\ell u}^{\Lambda}$ & $-\boldsymbol{\Lambda} / X^{\boldsymbol{\Lambda}}$ \\
\hline 5 & 12.45 & -0.0283 & 0.19 & 3.1303 & 10.41 & -0.0627 & 0.13 & 4.5077 & 9.16 & -0.1034 & 0.10 & 6.1354 \\
\hline 4 & 32.94 & 0.0098 & 0.70 & 1.6064 & 26.31 & -0.0078 & 0.49 & 2.3133 & 22.18 & -0.0287 & 0.36 & 3.1486 \\
\hline 3 & 63.38 & 0.0248 & 1.65 & 1.0062 & 49.97 & 0.0138 & 1.14 & 1.4490 & 41.32 & 0.0007 & 0.84 & 1.9722 \\
\hline
\end{tabular}

${ }^{\mathrm{c}}$ Parameter values: $\ell=-0.5 A L, x=-0.2 A L, u=-0.18 A L, \theta=0.25(\sigma=1 / 5), \theta=0.3(\sigma=1 / 6), \theta=0.35(\sigma=1 / 7), r=5 \%, P=10$.

because there is no expected gain assuming risks, and a positive probability of ruin remains. The purpose of Table 1 is to stress the idea that, in order to have a real chance of reducing the total expected discounted contributions at the expense of incurring an acceptable probability of ruin, we should be sure that, in the financial market, there is an asset with a high enough Sharpe ratio. Higher Sharpe ratios improve the performance of sensible management with respect to secure management, but the concrete size depends on the specific parameters defining the fund, and also on the risk exposure that the sponsor is willing to take. Notice also that the higher the reduction levels in liabilities are pursued, the higher the probability of ruin exposed by the fund. In the table, a 30\% reduction $(u=-0.14 A L)$ is also considered to show this fact more clearly.

Motivated by the table above, we consider a different range of admissible ruin probabilities for debt reduction of $5 \%$ and that for $10 \%$ and $20 \%$. Tables $2-4$ contain the following information: the probability of ruin, the percentage of the reduction in the total expected contribution, the value of $k$, the expected time for reaching the objectives in each case, and the ratio of investment in the risky asset with respect to the unfunded actuarial liability, $X$. This is provided for the three different Sharpe ratios selected. 
Table 4

Performance of sensible management with respect to secure management with debt reduction of $20 \%(\bar{t}=7.17)^{\mathrm{d}}$

\begin{tabular}{|c|c|c|c|c|c|c|c|c|c|c|c|c|}
\hline \multirow{3}{*}{$\begin{array}{l}\text { Probability } \\
\text { of ruin } \%\end{array}$} & \multicolumn{12}{|c|}{ Sharpe ratio } \\
\hline & \multicolumn{4}{|c|}{$\theta=0.25$} & \multicolumn{4}{|l|}{$\theta=0.30$} & \multicolumn{4}{|l|}{$\theta=0.35$} \\
\hline & $\frac{\mathbb{E} C^{k}(z)}{C^{k^{\prime}}(z)} \%$ & $k$ & $E_{x} \tau_{\ell u}^{\boldsymbol{\Lambda}}$ & $-\boldsymbol{\Lambda} / X^{\boldsymbol{\Lambda}}$ & $\frac{\mathbb{E} C^{k}(z)}{C^{k^{\prime}}(z)} \%$ & $k$ & $E_{x} \tau_{\ell u}^{\boldsymbol{\Lambda}}$ & $-\boldsymbol{\Lambda} / X^{\boldsymbol{\Lambda}}$ & $\frac{\mathbb{E} C^{k}(z)}{C^{k^{\prime}}(z)} \%$ & $k$ & $E_{x} \tau_{\ell u}^{\boldsymbol{\Lambda}}$ & $-\boldsymbol{\Lambda} / X^{\boldsymbol{\Lambda}}$ \\
\hline 5 & 74.47 & 0.0273 & 4.24 & 0.9087 & 59.31 & 0.0173 & 2.94 & 1.3085 & 49.21 & 0.0055 & 2.16 & 1.7810 \\
\hline 4 & 95.43 & 0.0316 & 6.07 & 0.7358 & 76.51 & 0.0235 & 4.22 & 1.0595 & 63.52 & 0.0139 & 3.10 & 1.4421 \\
\hline 3 & $>100$ & & & & 96.92 & 0.0286 & 5.98 & 0.8565 & 80.76 & 0.0209 & 4.39 & 1.1658 \\
\hline 2 & $>100$ & & & & $>100$ & & & & $>100$ & & & \\
\hline 1 & $>100$ & & & & $>100$ & & & & $>100$ & & & \\
\hline
\end{tabular}

d Parameter values: $\ell=-0.5 A L, x=-0.2 A L, u=-0.16 A L, \theta=0.25(\sigma=1 / 5), \theta=0.3(\sigma=1 / 6), \theta=0.35(\sigma=1 / 7), r=5 \%, P=10$.

\section{Comments on the results}

In Table 2, debt reduction of $5 \%$ is considered. We observe that, selecting $k<r$, the contribution can be reduced appreciably and, at the same time, the objective of debt elimination is attained with high probability and, of course, the results improve with higher Sharpe ratios and are worse in the reverse situation. To show an example, we can read in Table 2 that, with $\theta=0.3$, the expected contribution is roughly one half of the total contribution under inactive management, the expected time to eliminate $5 \%$ of the debt is 0.61 years (resp. 1.65), and the probability of ruin is of $1.5 \%$. A reduction in the expected contribution is possible thanks to the assumption of some risk of ruin by the sponsor, which allows greater flexibility in fund management. This is clearly shown in the trend observed for the coefficient $-\boldsymbol{\Lambda} / X^{\Lambda}$, which increases with the probability of ruin and, as can be seen in Tables 3 and 4 , decreases with the debt reduction target.

More ambitious debt reduction goals imply amortization efforts that, being small still than under secure management, are not as impressive as with a 5\% objective. Taking on more risks raises the probability of ruin, but drastically reduces the total contribution effort. When the objective of debt reduction is greater (10\% and $20 \%)$, investment behavior is more cautious, as shown by the fact that the amount invested in the risky asset is proportionally smaller. Note that, in order to attain small probabilities of ruin in this case, the expected contribution must be greater than the contribution in the secure case, hence sensible investment makes sense only if, in fact, the sponsor is willing to take some positive risk. The behavior of the expected times to reach the objectives follows a pattern similar to the expected contributions, increasing with $k$ and with debt reduction, and decreasing with the Sharpe ratio.

Also note that, in some cases, the parameter $k$ becomes negative, indicating that the contribution is below the normal cost, even when the fund is underfunded.

Our general comments are that the way sensible management compares with secure management depends highly on the expected return of the portfolio by the unit of risk and of the level of reduction in the unfunded actuarial liability that is pursued. For reasonable values of Sharpe ratio and moderate values of reduction debt, risk investment leads to the attainment of the objective with high probability, a small expected time, and an appreciable reduction in the amortization effort.

\subsection{Overfunded region, $u>0$}

In this case, the objective is to maximize the probability of reaching $u$ before some value $\ell$, with $0<\ell<u$. In contradistinction with the underfunded case, to attain this objective in an optimal way, the parameter $k$ must be chosen to be greater than $r$. In fact, when $k \leq r$, the problem is not a genuine stochastic problem, because no investment in risky assets assures that the fund increases to $u(k<r)$ or remains constant $(k=r)$.

Proposition 3.2. Assume that $k>r$ and $0<\ell<x<u$. The optimal investment strategy $\boldsymbol{\Lambda}_{U}(x)$ and the value function are given by (10) and (11), respectively, with the parameter $\alpha$ as was defined in Proposition 3.1, whenever $\alpha \neq 0$. They are given by

$$
\boldsymbol{\Lambda}_{U}(X)=\Sigma^{-1}(\mathbf{b}-r \mathbf{1}) X=\Sigma^{-1}(\mathbf{b}-r \mathbf{1})(A L-F)
$$


and

$$
U(x)=\frac{\ln x-\ln \ell}{\ln u-\ln \ell}=\frac{\ln (A L-F)-\ln \ell}{\ln u-\ln \ell},
$$

respectively, when $\alpha=0$.

Similar remarks apply to this result as those that were applied to Proposition 3.1, some of the differences now being that $\alpha<0$ when $r<k<r+\theta^{\top} \theta / 2, \alpha=0$ when $k=r+\theta^{\top} \theta / 2$, and $0<\alpha<1$ when $k>r+\theta^{\top} \theta / 2$. In the underfunded case, only $\alpha>1$ appears, which is equivalent to $k<r$.

As in the underfunded case, the total discounted value of the expected contributions under the optimal solution (which implies sensible management) and under secure management could be compared. The conclusions would be very similar to that situation, the more important one being that the expected contribution is smaller in the former case than in the latter case, in compensation for taking on more risk in the management, that is, a positive but small probability of ruin.

\section{Minimizing/maximizing the expected discounted penalty/reward}

The ruin point is the level of wealth in the fund that is considered by the sponsor as the minimum floor admissible for continuing financial operations with a certain guarantee of attending the present and future liabilities. As we have shown in the previous section, the probability of ruin can be kept to a minimum and, at the same time, total expected discounted contributions reduced, by selecting the spread constant $k$ in the amortization scheme below the risk-free interest rate, $r$. The question that we address in this section is how this ruin point can be made more tangible, in the sense that, once the fund has fallen to this undesirable value, the fund is really penalized - maybe due to the fact that a great injection of capital is demanded by law, in order to raise cash into the fund account. Hence, we now consider the existence of a constant penalization, measured in monetary units, incurred by the pension fund if the fund assets fall to the ruin value $\ell$ defined in the section above. We suppose, without lost of generality, that the penalization is one unit. The value of the penalization today is $\mathrm{e}^{-\mu \tau_{\ell}^{\Lambda}}$, where $\mu>0$ is the subjective discount factor of the sponsor valuing the penalty, hence the objective is to minimize $\mathbb{E}\left(\mathrm{e}^{-\mu \tau_{\ell}^{\Lambda}}\right)$, the expected discounted disutility when hitting $\ell$. We define $G_{\ell}(x)=\inf _{\boldsymbol{\Lambda} \in \mathcal{A}_{x}} \mathbb{E}_{x}\left(\mathrm{e}^{-\mu \tau_{\ell}^{\Lambda}}\right)$, with $\ell<x$, with associated optimal policy $\boldsymbol{\Lambda}_{G_{\ell}}$, i.e. $\boldsymbol{\Lambda}_{G_{\ell}}(x)=\arg \inf \boldsymbol{\Lambda}_{\boldsymbol{A}} \mathcal{A}_{x} \mathbb{E}_{x}\left(\mathrm{e}^{-\mu \tau_{\ell}^{\Lambda}}\right)$. A parallel problem to the one just stated is that of maximizing the expected discounted value of attaining a prescribed higher value of the fund. We are thinking of the possibility that the sponsor can be rewarded due to good management, driving the fund to an upper level, $u$. The problem now becomes that of maximizing the expected discounted value of the reward to the present time. So, we define $G_{u}(x)=\sup _{\Lambda \in \mathcal{A}_{x}} \mathbb{E}_{x}\left(\mathrm{e}^{-\mu \tau_{u}^{\Lambda}}\right), x<u$, with the associated optimal strategy $\boldsymbol{\Lambda}_{G_{u}}$, i.e. $\boldsymbol{\Lambda}_{G_{u}}(x)=\arg \sup _{\boldsymbol{\Lambda} \in \mathcal{A}_{x}} \mathbb{E}_{x}\left(\mathrm{e}^{-\mu \tau_{u}^{\Lambda}}\right)$. As in the previous section, when the fund is underfunded and we want to avoid punishment, only the selection $k<r$ makes the question interesting of how to manage the fund, because $k \geq r$ guarantees that full investment in the bond avoids the ruin point for sure. To unify the analysis, we consider only $k<r$ in the overfunded case. In fact, when $k>r$, the interest charged to the surplus $X$ is negative (see the paragraph after Eq. (9)), which is in some sense in contradiction to the aim of attaining a reward due to high values of $X$. This causes some problems in the resolution of this case.

As we can see in the following proposition, the investment pattern associated with minimizing the expected discounted penalization and maximizing the expected discounted reward has the same structure to that of the above section, that is, it is of constant proportionality type with respect to the liabilities.

Proposition 4.1. The optimal investment strategies and the optimal value functions are, respectively,

(i) (Underfunded region). Assume that $\ell<x<0$ and $k<r$. Then the optimal investment policy is given by

$$
\boldsymbol{\Lambda}_{G_{\ell}}(X)=-\frac{1}{q^{+}-1} \Sigma^{-1}(\mathbf{b}-r \mathbf{1}) X=\frac{1}{q^{+}-1} \Sigma^{-1}(\mathbf{b}-r \mathbf{1})(A L-F),
$$

and the minimum penalization cost is given by

$$
G_{\ell}(x)=\left(\frac{x}{\ell}\right)^{q^{+}}=\left(\frac{F-A L}{\ell}\right)^{q^{+}},
$$


where

$$
q^{+}=\frac{1}{2(r-k)}\left(r-k+\frac{1}{2} \theta^{\top} \theta+\mu+\sqrt{\Phi}\right)
$$

with

$$
\Phi=\left(r-k+\frac{1}{2} \theta^{\top} \theta+\mu\right)^{2}-4(r-k) \mu .
$$

(ii) (Overfunded region). Suppose that $0<x<u$ and $k<r$. Then the optimal investment strategy maximizing the premium is given by

$$
\boldsymbol{\Lambda}_{G_{u}}(X)=\frac{1}{1-q^{-}} \Sigma^{-1}(\mathbf{b}-r \mathbf{1}) X=\frac{1}{1-q^{-}} \Sigma^{-1}(\mathbf{b}-r \mathbf{1})(F-A L),
$$

and the maximum premium is given by

$$
G_{u}(x)=\left(\frac{x}{u}\right)^{q^{-}}=\left(\frac{F-A L}{u}\right)^{q^{-}},
$$

where

$$
q^{-}=\frac{1}{2(r-k)}\left(r-k+\frac{1}{2} \theta^{\top} \theta+\mu-\sqrt{\Phi}\right) .
$$

Remark 4.1. In the case $k=r$, it can be checked, following the proof of Proposition 4.1, that the optimal investment and the value function are

$$
\begin{aligned}
& \boldsymbol{\Lambda}_{G_{u}}(X)=\frac{1}{1-q} \Sigma^{-1}(\mathbf{b}-r \mathbf{1}) X=\frac{1}{1-q} \Sigma^{-1}(\mathbf{b}-r \mathbf{1})(F-A L), \\
& G_{u}(x)=\left(\frac{x}{u}\right)^{q}=\left(\frac{F-A L}{u}\right)^{q},
\end{aligned}
$$

respectively, with

$$
q=\frac{\mu}{\mu+\theta^{\top} \theta / 2} \in(0,1),
$$

being precisely the limits of the expressions (15) and (16) when $k$ goes to $r$.

Remark 4.2. To determine the regions of borrowing and short-selling in each case, notice that the assumption $k<r$ imposed in Proposition 4.1 implies that $0<q^{-}<1<q^{+}$. Suppose first that the aim is to minimize the penalization inherent to the drop in fund assets to $\ell$ when the fund is underfunded. Then it is optimal borrowing at rate $r$ to invest in asset $i, \mu_{i, G_{\ell}} \geq F$, if and only if $F \leq v_{i}^{\prime} A L$, with $v_{i}^{\prime}=\mathbf{e}_{i} \Sigma^{-1}(\mathbf{b}-r \mathbf{1}) /\left(\mathbf{e}_{i} \Sigma^{-1}(\mathbf{b}-r \mathbf{1})+q^{+}-1\right)$. In the same way, it is optimal borrowing to invest in some stock, $\sum_{i=1}^{n} \mu_{i, G_{\ell}} \geq F$, if and only if $F \leq v_{0}^{\prime} A L$, with $v_{0}^{\prime}=\mathbf{1}^{\top} \Sigma^{-1}(\mathbf{b}-r \mathbf{1}) /\left(\mathbf{1}^{\top} \Sigma^{-1}(\mathbf{b}-r \mathbf{1})+q^{+}-1\right)$. Suppose now that the objective is to maximize the expected payoff associated with driving the fund to $u$ in the overfunded region. In this case, the borrowing region is determined as follows. Substituting $q^{+}$by $q^{-}$in the corresponding expressions, the inequalities affecting $v_{i}^{\prime}$ are reversed and the denominators appearing in the expressions for $v_{i}^{\prime}$ can be negative, i.e. $\mathbf{e}_{i} \Sigma^{-1}(\mathbf{b}-r \mathbf{1}) \leq 1-q^{-}$. In that case, borrowing is not optimal, because $\mu_{i, G_{u}} \leq F-A L<F$. Finally, in both cases the optimal strategies are positive, thus short-selling is not optimal.

Remark 4.3. Taking the limit as the discount factor $\mu$ goes to 0 , it is possible to recover from Proposition 4.1 the solution of the problem of maximizing the probability that the fund assets reach the level $u$ before $\ell$, explored in the previous section. Substituting the strategy $\boldsymbol{\Lambda}_{G_{\ell}}$ of (12) into the fund equation (9), we obtain that the process $X$ satisfies the stochastic differential equation

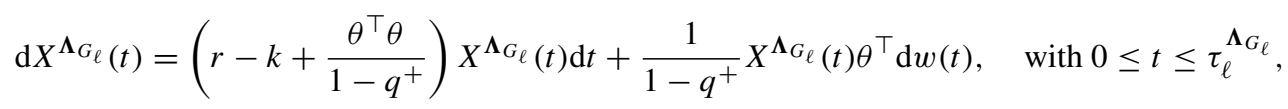


which is a geometric Brownian motion. It is very easy to check that

$$
G_{\ell}\left(X^{\left.\boldsymbol{\Lambda}_{G_{\ell}}(t)\right)}=G_{\ell}(x) \exp \left\{\left(r-k+\frac{\theta^{\top} \theta}{1-q^{+}}-\frac{\theta^{\top} \theta}{2\left(1-q^{+}\right)^{2}}\right) q^{+} t+\frac{q^{+}}{1-q^{+}} \theta^{\top} w(t)\right\},\right.
$$

for $0 \leq t \leq \tau_{\ell}^{\boldsymbol{\Lambda}_{G_{\ell}}}$, and from this we obtain ${ }^{4}$

$$
\mathbb{E}_{x}\left(\tau_{\ell}^{\boldsymbol{\Lambda}_{G_{\ell}}}\right)=\frac{\ln |\ell|-\ln |x|}{r-k+\frac{\theta^{\top} \theta}{1-q^{+}}-\frac{\theta^{\top} \theta}{2\left(1-q^{+}\right)^{2}}},
$$

depending on $\mu$ through $q^{+}$.

When the problem is to maximize rewards, analogous comments apply, but now substituting adequately $q^{+}$by $q^{-}$, and $\ell$ by $u$, in the corresponding expressions.

Letting the discount factor $\mu$ go to zero, the root $q^{+}$converges to $\alpha$ (this constant was defined in Proposition 3.1) because $k<r$ (observe that $\alpha, q^{+}>1$ ). The root $q^{-}$converges to 0 , and root $q$ (defined when $k=r$ ) converges to 0 . These facts imply the following convergences:

$$
\lim _{\mu \rightarrow 0} \boldsymbol{\Lambda}_{G_{\ell}}=\Lambda_{U}, \quad \lim _{\mu \rightarrow 0} G_{\ell}(x)=\left(\frac{x}{\ell}\right)^{\alpha},
$$

if $k<r$; and

$$
\lim _{\mu \rightarrow 0} \boldsymbol{\Lambda}_{G_{u}}(X)=\Sigma^{-1}(\mathbf{b}-r \mathbf{1}) X=\Sigma^{-1}(\mathbf{b}-r \mathbf{1})(F-A L), \quad \lim _{\mu \rightarrow 0} G_{u}(x)=1,
$$

if $k \leq r$.

To finish this section, the following proposition shows that, in the overfunded region, the strategy appearing in (20) solves the problem of minimizing the expected time of reaching $u$ before $\ell$. So we will consider $0<\ell<x<u$. Let $V_{u}(x)=\inf _{\boldsymbol{\Lambda} \in \mathcal{A}_{x}} \mathbb{E}_{x}\left(\tau_{u}^{\boldsymbol{\Lambda}}\right)$ be the value function and $\boldsymbol{\Lambda}_{V_{u}}(x)=\arg \inf _{\boldsymbol{\Lambda} \in \mathcal{A}_{x}} \mathbb{E}_{x}\left(\tau_{u}^{\boldsymbol{\Lambda}}\right)$ the optimal investment.

Proposition 4.2. If $0<x<u$ and $k \leq r$, then the optimal investment strategy is given by

$$
\boldsymbol{\Lambda}_{V_{u}}(X)=\Sigma^{-1}(\mathbf{b}-r \mathbf{1}) X=\Sigma^{-1}(\mathbf{b}-r \mathbf{1})(F-A L)
$$

and the value function by

$$
V_{u}(x)=\frac{\ln u-\ln x}{r-k+\frac{1}{2} \theta^{\top} \theta}=\frac{\ln u-\ln (F-A L)}{r-k+\frac{1}{2} \theta^{\top} \theta} .
$$

As $\mu$ is positive, $q^{+}<\alpha$; so the strategy minimizing penalization in the underfunded case $(k<r)$ involves less risky investment than the policy maximizing the probability of hitting $u$ before $\ell, \ell<u$, that is, $\boldsymbol{\Lambda}_{G_{\ell}}<\boldsymbol{\Lambda}_{U}$.

\section{Optimal policies under risk of sudden termination}

Up to now, we have been supposing that the pension plan under consideration stands forever, that is, there is no known, fixed time of termination of the funding process. This is because the plan is of aggregated type and the retirement of the participants takes place continuously at different times. In this way, the plan covers the obligations at all times and this process continues for all the new entrants in the fund. Consider, however, that there is some positive probability of bankruptcy of the sponsoring firm. We are not concerned with the situation of beneficiaries and insurance companies, but with the issue of optimal management of the fund in these circumstances until the time of termination. To model such a possibility, let us consider that the distribution function of the time $T$ of termination is

\footnotetext{
${ }^{4}$ Notice that (18) could not be obtained from the generating function of $\tau_{\ell}{ }_{G_{\ell}}$. The property that if $\varphi(\mu)=\mathbb{E}\left(\mathrm{e}^{-\mu Z}\right)$, with $\mu \geq 0$ and where $Z$ is a random variable, then $\varphi(0)=\mathbb{P}(Z<\infty)$ and $-\varphi^{\prime}(0)=\mathbb{E} Z$ cannot be applied here because $\tau_{\ell} \boldsymbol{\Lambda}_{G_{\ell}}$ also depends on $\mu$.
} 
of exponential form, i.e. $\mathbb{P}(T \leq t) \equiv h(t)=1-\mathrm{e}^{-\rho t}$ is the probability that sudden termination does not occur until time $t \geq 0$, where $\rho>0$.

Let $L$ be an instantaneous utility function, depending on the process $X=F-A L$. The objective of the sponsor will be to maximize (note that we identify the random variable $T$ with their values)

$$
\begin{aligned}
\mathbb{E}\left(\mathbb{E}_{x} \int_{0}^{T} L(X(t)) \mathrm{d} t\right) & =\int_{0}^{\infty} \mathbb{E}_{x}\left(\int_{0}^{T} L(X(t)) \mathrm{d} t\right) h^{\prime}(T) \mathrm{d} T \\
& =\mathbb{E}_{x} \int_{0}^{\infty} L(X(t))\left(\int_{t}^{\infty} h^{\prime}(T) \mathrm{d} T\right) \mathrm{d} t \\
& =\mathbb{E}_{x} \int_{0}^{\infty} \mathrm{e}^{-\rho t} L(X(t)) \mathrm{d} t
\end{aligned}
$$

where we have used independence between $T$ and $w$, applied Fubini in the second equality, and integrated by parts in the third. ${ }^{5}$

The objective functional can be given a different meaning: $\mathrm{e}^{-\rho t}$ is a factor weighing the instantaneous utility at time $t$. Thus $\rho$ can be seen as a rate of impatience of the controller or as an actualization rate. In this interpretation, the fund is considered to stay forever.

The objective functional of the sponsor is thus ${ }^{6} \mathbb{E}_{x} \int_{0}^{\infty} \mathrm{e}^{-\rho t} L(X(t)) \mathrm{d} t$, where the process $X(t)$ is constrained by (9). Of course, function $L$ must show different properties in the underfunded region and in the overfunded region, in accordance with the priority of the manager in each case. When $F<A L$, i.e. $x<0$, the sponsor penalizes deviations of the fund with respect to $A L$. In contrast, when $F>A L$, i.e. $x>0$, the sponsor obtains utility from the surplus. As $F$ increases from $A L$, the contribution rate decreases, making it possible to become negative, meaning, in this case, that the firm obtains dividends from the fund assets. Therefore, an adequate selection in the underfunded case seems to be the convex function $L(X)=|X|^{\gamma} / \gamma$, with $\gamma>1$, whereas in the overfunded region we suppose the concave utility specification $L(X)=X^{\gamma} / \gamma$, with $\gamma<1$ and $\gamma \neq 0$, or $L(X)=\ln X$. Let us denote by $W$ the value function associated with the problem. In the first case the objective is to minimize and in the second case the objective is to maximize.

Other authors have considered models where the objective is to maximize a terminal utility, depending on the contribution rate instead of the fund and with a final time $T$ fixed previously; see Boulier et al. (1995), Siegmann and Lucas (1999) or Cairns (2000).

For the maximization case, following Fleming and Soner (1993), the HJB equation for the problem is

$$
-\rho V(x)+\sup _{\boldsymbol{\Lambda}}\left\{\left((r-k) x+\boldsymbol{\Lambda}^{\top}(\mathbf{b}-r \mathbf{1})\right) V^{\prime}(x)+\frac{1}{2} \boldsymbol{\Lambda}^{\top} \Sigma \boldsymbol{\Lambda} V^{\prime \prime}(x)+L(x)\right\}=0 .
$$

Supposing that $V^{\prime \prime}<0$, the maximizer is again as in (28). Substituting this in the equation above, we have

$$
-\rho V(x)+(r-k) x V^{\prime}(x)-\frac{1}{2} \theta^{\top} \theta \frac{\left(V^{\prime}(x)\right)^{2}}{V^{\prime \prime}(x)}+L(x)=0 .
$$

An analogous equation is obtained for the minimization case, substituting sup by inf.

For the following result, define the constants $\xi, \eta$ by

$$
\begin{aligned}
\xi & \equiv \frac{1}{\rho+\frac{\theta^{\top} \theta}{2} \frac{\gamma}{\gamma-1}-\gamma(r-k)}, \\
\eta & \equiv \frac{r-k+\frac{\theta^{\top} \theta}{2}}{\rho^{2}} .
\end{aligned}
$$

\footnotetext{
5 This derivation is well known and is introduced here only for completeness.

6 Notice that the stated problem is not exactly the model in Merton (1971), since that utility depends on the controlled process $X$ and not on the control variable as in Merton's model (the consumption process in that case). Hence the optimality proof needs Proposition 5.1.
} 
Table 5

Optimal investment decisions in the different cases studied

Minimizing probability of ruin

Minimizing penalty

Maximizing reward

Minimizing time

Uncertain time horizon

$$
\begin{aligned}
& \boldsymbol{\Lambda}_{U}(X)=-\frac{2(r-k)}{\theta^{\top} \theta} \Sigma^{-1}(\mathbf{b}-r \mathbf{1}) X \\
& \boldsymbol{\Lambda}_{G_{\ell}}(X)=-\frac{1}{q^{+}-1} \Sigma^{-1}(\mathbf{b}-r \mathbf{1}) X \\
& \boldsymbol{\Lambda}_{G_{u}}(X)=-\frac{1}{q^{-}-1} \Sigma^{-1}(\mathbf{b}-r \mathbf{1}) X \\
& \boldsymbol{\Lambda}_{V_{u}}(X)=\Sigma^{-1}(\mathbf{b}-r \mathbf{1}) X \\
& \boldsymbol{\Lambda}_{W}(X)=-\frac{1}{\gamma-1} \Sigma^{-1}(\mathbf{b}-r \mathbf{1}) X
\end{aligned}
$$

Table 6

Comparison of the optimal investment decisions

\section{Underfunded case}

$\boldsymbol{\Lambda}_{U}>\boldsymbol{\Lambda}_{W} \Leftrightarrow k>r-\frac{\theta^{\top} \theta}{2(\gamma-1)}$

$\boldsymbol{\Lambda}_{U}=\boldsymbol{\Lambda}_{W} \Leftrightarrow k=r-\frac{\theta^{\top} \theta}{2(\gamma-1)}$

$\boldsymbol{\Lambda}_{G_{\ell}}>\boldsymbol{\Lambda}_{W} \Leftrightarrow q^{+}>\gamma$

$\boldsymbol{\Lambda}_{G_{\ell}}=\boldsymbol{\Lambda}_{W} \Leftrightarrow q^{+}=\gamma$
Overfunded case

$\boldsymbol{\Lambda}_{U}>\boldsymbol{\Lambda}_{W} \Leftrightarrow k>r-\frac{\theta^{\top} \theta}{2(\gamma-1)}$
$\boldsymbol{\Lambda}_{U}=\boldsymbol{\Lambda}_{W} \Leftrightarrow k=r-\frac{\theta^{\top} \theta}{2(\gamma-1)}$
$\boldsymbol{\Lambda}_{G_{u}}>\boldsymbol{\Lambda}_{W} \Leftrightarrow q^{-}, q>\gamma$
$\boldsymbol{\Lambda}_{G_{u}}=\boldsymbol{\Lambda}_{W} \Leftrightarrow q^{-}, q=\gamma$
$\boldsymbol{\Lambda}_{W}>\boldsymbol{\Lambda}_{V_{u}} \Leftrightarrow \gamma>0$
$\boldsymbol{\Lambda}_{W}=\boldsymbol{\Lambda}_{V_{u}} \Leftrightarrow \gamma=0$

Proposition 5.1. The optimal investment strategy and optimal value functions are, respectively:

(i) If $L(X)=|X|^{\gamma} / \gamma$ with $\gamma>1$ or if $L(X)=X^{\gamma} / \gamma$ with $\gamma<1$ and in both cases $\xi>0$, then

$$
\mathbf{\Lambda}_{W}(X)=-\frac{1}{\gamma-1} \Sigma^{-1}(\mathbf{b}-r \mathbf{1}) X=-\frac{1}{\gamma-1} \Sigma^{-1}(\mathbf{b}-r \mathbf{1})(F-A L),
$$

and the value function is $W(x)=\xi|x|^{\gamma} / \gamma=\xi|F-A L|^{\gamma} / \gamma$.

(ii) If $L(X)=\ln X$, then

$$
\boldsymbol{\Lambda}_{W}(X)=\Sigma^{-1}(\mathbf{b}-r \mathbf{1}) X=\Sigma^{-1}(\mathbf{b}-r \mathbf{1})(F-A L),
$$

and the value function is $W(x)=(\ln x) / \rho+\eta=(\ln (F-A L)) / \rho+\eta$.

Remark 5.1. Note that the optimal policy (26) is also optimal for the problem solved in Proposition 3.2 in the overfunded case with $\alpha=0$. On the other hand, if $r-\theta^{\top} \theta /(2(\gamma-1))>0$, then, choosing a spread method of contribution with $k=r-\theta^{\top} \theta /(2(\gamma-1))$, we have that (25) and (10) coincide in the underfunded case. An analogous conclusion holds in the overfunded region. In a similar way, we can compare (25) with (12) and (15). Hence the problem of maximizing/minimizing the reward/penalty/time to hit a goal and the problem of minimizing the probability of ruin have the same optimal solution as the problem of maximizing/minimizing the terminal utility with an adequate selection of the parameter $k$. Table 5 shows which are the optimal investment policies in each case, whereas Table 6 compares the size of the respective optimal investments. In these tables, other interesting cases are included.

\section{Conclusions}

We have analyzed the management of a pension funding process of an aggregated pension plan in three different situations, distinguishing between the overfunded and the underfunded cases. First, we have supposed that the aim of the employer is to maximize the probability of avoiding a ruin point. Second, we have studied the problems of minimizing the cost of beating a lower barrier and maximising the prize of reaching an upper bound, and finally we have considered the maximization of some utility along an infinite horizon, when taking into account the possibility of exogenous shocks leading the pension plan to sudden termination.

A key conclusion of the paper is that the optimal investment policies in all cases reported are of proportional type with respect to the gap between the fund assets and the actuarial liability. This is a direct consequence of the choices 
of objective functions. It is worth noting that this investment behavior is also optimal in the model of Merton of consumption and portfolio choice with i.i.d. returns and HARA utility. The vector $\Sigma^{-1}(\mathbf{b}-r \mathbf{1})$, appearing as one of the components of the proportionality vector in the solutions shown in Table 5, is called by Merton the optimalgrowth portfolio strategy. It has the property of maximizing the expected logarithm utility of terminal wealth and the expected continuously compounded return. Since the objectives of the sponsor are related to the growth of fund assets - which turns out to be equivalent to minimizing the probability of ruin in Section 3 - it is not a surprise that the optimal-growth portfolio strategy appears as an integral part of the proportional investment strategy.

Proportional investment strategies, independent of the deficit level, leads in Section 3 to the apparent paradoxical behavior of taking on more risk as the deficit increases. The justification is that the return obtained with safe investment plus amortization effort are not sufficient to cover the liabilities — recall that we are supposing that $k<r$. Hence, it is necessary to invest in risky assets in the underfunded region in order to obtain higher mean returns, so the uncertain effects are always present. When the fund is near the ruin point, it becomes optimal to augment the mean returns of the fund assets, assuming the risk of a higher volatility. The situation is similar in Proposition 4.1, where, due to the proportional pattern in the investment behavior, investment in equities increases as the deficit or surplus increases.

Another interesting property is that the optimal investment strategy does not depend on the ruin point $\ell$ and the desired level $u$. For the class of funds described in this paper and with the objectives proposed, there is no difference in their optimal management, as long as they differ only in the ruin level considered. However, the amortization effort $k$ has a major impact, not only in the optimal investment decisions but also in the probability of ruin, the expected time to attain the objectives, and the total amount of expected contributions made to the fund.

Further research should be directed to including stochastic benefits as in Josa-Fombellida and Rincón-Zapatero (2004). In this case, there are two sources of uncertainty: the returns of fund assets and the evolution of benefits. This leads to a more difficult problem, where it is not possible to obtain explicit solutions, except in very particular cases. Thus the analysis must be based on proving the existence and uniqueness of the solution to the HJB equation from a more theoretical point of view. It may be appropriate to study this problem in the framework of the viscosity theory of PDEs, analyzing the qualitative properties of the optimal solution.

\section{Acknowledgements}

We are grateful to an anonymous referee for careful reading of the paper and helpful comments and suggestions. Both authors acknowledge financial support from Consejería de Educación y Cultura de la Junta de Castilla y León (Spain) under project VA099/04 and Spanish Ministerio de Ciencia y Tecnología and FEDER funds under project BFM2002-00425.

\section{Appendix A}

The survival and growth problems that we consider in this paper are particular cases of the most general optimal stopping control problem that we present below. Given an investment policy $\boldsymbol{\Lambda}$, we denote by $\tau_{z}^{\Lambda}$ the first hitting time to value $z$ of the process $X: \tau_{z}^{\Lambda}=\inf \left\{t>0: X^{\Lambda}(t)=z\right\}$. Given $\ell, u$, with $\ell<X(0)=x<u, \tau_{\ell u}^{\Lambda}$ is the first escape time from interval $(\ell, u)$ of the process $X$ under policy $\Lambda: \tau_{\ell u}^{\Lambda}=\min \left\{\tau_{\ell}^{\Lambda}, \tau_{u}^{\Lambda}\right\}$.

For the continuous functions $g$ and $h$, with $g$ bounded, the nonnegative function $\lambda$, and given $\ell, u$, the objective functional is

$$
J(x, \boldsymbol{\Lambda})=\mathbb{E}_{x}\left(\int_{0}^{\tau_{\ell u}^{\Lambda}} g\left(X^{\boldsymbol{\Lambda}}(t)\right) \mathrm{e}^{-\int_{0}^{t} \lambda\left(X^{\boldsymbol{\Lambda}}(s)\right) \mathrm{d} s} \mathrm{~d} t+h\left(X^{\boldsymbol{\Lambda}}\left(\tau_{\ell u}^{\boldsymbol{\Lambda}}\right)\right) \mathrm{e}^{-\int_{0}^{\tau_{\ell u}^{\Lambda}} \lambda\left(X^{\boldsymbol{\Lambda}}(s)\right) \mathrm{d} s}\right),
$$

the value function is $\widehat{V}(x)=\sup _{\boldsymbol{\Lambda} \in \mathcal{A}_{x}}\{J(x, \boldsymbol{\Lambda}):$ s.t. (9) $\}$ and the optimal investment policy is $\boldsymbol{\Lambda}_{\widehat{V}}(x)=$ $\arg \sup _{\boldsymbol{\Lambda} \in \mathcal{A}_{x}}\{J(x, \boldsymbol{\Lambda}):$ s.t. (9) $\}$, where $\mathcal{A}_{x}=\{\boldsymbol{\Lambda}: J(x, \boldsymbol{\Lambda})<\infty\}$ is the set of adapted and admissible controls given $X(0)=x$.

The Hamilton-Jacobi-Bellman (HJB henceforth) equation associated with this problem is (see Krylov, 1980):

$$
\sup _{\boldsymbol{\Lambda}}\left\{\left((r-k) x+\boldsymbol{\Lambda}^{\top}(\mathbf{b}-r \mathbf{1})\right) V^{\prime}(x)+\frac{1}{2} \boldsymbol{\Lambda}^{\top} \Sigma \boldsymbol{\Lambda} V^{\prime \prime}(x)-\lambda(x) V(x)+g(x)\right\}=0,
$$


subject to the boundary conditions $V(\ell)=h(\ell)$ and $V(u)=h(u)$. If $V$ is sufficiently regular and strictly concave, the argument maximizing (27) is

$$
\widehat{\boldsymbol{\Lambda}}\left(V^{\prime}(x), V^{\prime \prime}(x)\right)=-\Sigma^{-1}(\mathbf{b}-r \mathbf{1}) \frac{V^{\prime}(x)}{V^{\prime \prime}(x)} .
$$

By substituting (28) in (27), we obtain

$$
(r-k) x V^{\prime}(x)-\frac{1}{2} \theta^{\top} \theta \frac{\left(V^{\prime}(x)\right)^{2}}{V^{\prime \prime}(x)}-\lambda(x) V(x)+g(x)=0, \quad \ell<x<u,
$$

with

$$
V(\ell)=h(\ell) \quad \text { and } \quad V(u)=h(u) .
$$

The following result, which is proved in more general versions in Browne (1997, 1999), shows that, under some conditions, the value function is characterized as the solution of (29)-(30).

Theorem A.1. Suppose that $V:(\ell, u) \longrightarrow(-\infty, \infty)$ of class $\mathcal{C}^{2}$, increasing and concave, that is to say, $V^{\prime}>0$ and $V^{\prime \prime}<0$, is the solution of the nonlinear Dirichlet equation (29) and (30) and satisfies the conditions:

(i) $\left(V^{\prime}\right)^{2} / V^{\prime \prime}$ is bounded in $(\ell, u)$;

(ii) either there exists an integrable random variable $Y$ such that $V\left(X^{\boldsymbol{\Lambda}}(t)\right) \geq Y$, for all $t \geq 0$ and for all $\boldsymbol{\Lambda} \in \mathcal{A}_{x}$, or

$$
\mathbb{E} \int_{0}^{t}\left(X^{\boldsymbol{\Lambda}}(s) V^{\prime}\left(X^{\boldsymbol{\Lambda}}(s)\right)\right)^{2} \sigma \boldsymbol{\Lambda}^{\top}(s) \boldsymbol{\Lambda}(s) \sigma^{\top} \mathrm{d} s<\infty,
$$

for every $t \geq 0$ and for every $\Lambda \in \mathcal{A}_{x}$;

(iii) $V^{\prime} / V^{\prime \prime}$ is locally Lipschitz continuous.

Then, $V$ is the optimal value function, i.e. $\widehat{V}=V$, and moreover the vector of optimal investment amounts is

$$
\mathbf{\Lambda}_{V}(x)=-\Sigma^{-1}(\mathbf{b}-r \mathbf{1}) \frac{V^{\prime}(x)}{V^{\prime \prime}(x)}, \quad \ell<x<u .
$$

\section{Appendix B}

Proof of Proposition 3.1. Applying Theorem A.1 with $g=\lambda=0, h(l)=0$ and $h(u)=1$, the optimal value function $U$ must be the increasing and concave solution of

$$
(r-k) x U^{\prime}(x)-\frac{1}{2} \theta^{\top} \theta \frac{\left(U^{\prime}(x)\right)^{2}}{U^{\prime \prime}(x)}=0, \quad l<x<u,
$$

with $U(l)=0$ and $U(u)=1$. The general solution of this ordinary differential equation is $U(x)=a_{1}+a_{2}|x|^{\alpha}$. The parameter $\alpha$ turns out to be $\alpha=1+\theta^{\top} \theta /(2(r-k))>1$. The boundary conditions determine the constants $a_{1}$ and $a_{2}$, and therefore the optimal value function is given by (11). It is immediate to check that $U^{\prime}>0$ and $U^{\prime \prime}<0$ and that the remaining hypotheses in Theorem A.1 hold. For values of $k$ greater than $r$, the solution obtained is not concave, hence the HJB equation is not maximized. Thus the problem as it stands, that is, without constraints on the decision variables, has no solution.

Proof of Proposition 3.2. As in the proof of Proposition 3.1 above, the general solution when $\alpha \neq 0$ is $U(x)=$ $a_{1}+a_{2} x^{\alpha}$. In this case, $\alpha<1$ because $k>r$. The frontier conditions allow one to obtain $a_{1}$ and $a_{2}$, in particular $a_{2}=1 /\left(u^{\alpha}-\ell^{\alpha}\right)$. Function $U$ is increasing and concave, because $a_{2} \alpha>0$. As in the previous proposition, it is immediate that (10) and (11) are the vector of optimal controls and the value function. For $k<r, U$ is not concave, hence in this case the HJB equation is not maximized and thus the problem has no solution. In the case $\alpha=0$, the general solution is $U(x)=a_{1}^{0}+a_{2}^{0} \ln x$, with constants $a_{1}^{0}$ and $a_{2}^{0}$ determined by the boundary conditions. It turns out that this solution is increasing and concave. 
Proof of Proposition 4.1. (i) Let us suppose that $\ell<x<0$ and $k<r$. Theorem A.1 is valid for minimization if we change sup by inf in the corresponding HJB equation and if the solution $V$ is strictly convex and decreasing. Function $G_{\ell}$ must be a strictly convex and decreasing solution of

$$
(r-k) V^{\prime}(x)-\frac{1}{2} \theta^{\top} \theta \frac{\left(V^{\prime}(x)\right)^{2}}{V^{\prime \prime}(x)}-\mu V(x)=0, \quad \ell<x<0,
$$

with $G_{\ell}(\ell)=1$ and $\boldsymbol{\Lambda}_{G_{\ell}}$ is obtained from (31).

This partial differential equation admits the solution $V(x)=p(-x)^{q}, \ell<x<0$, where $q$ is a root of the quadratic equation

$$
(r-k) q^{2}-\left(r-k+\theta^{\top} \theta / 2+\mu\right) q+\mu=0
$$

and $p$ is a constant to be determined from the boundary condition, that is to say $p=|\ell|^{-q}$. The two solutions (14) and (17) are obtained from (33). Because $k<r$, it is not difficult to prove that the discriminant $\Phi$ of Eq. (33) is positive and that $0<q^{-}<1<q^{+}$. On the other hand, $G_{\ell}$ is decreasing $\left(G_{\ell}^{\prime}<0\right)$ and convex $\left(G_{\ell}^{\prime \prime}<0\right)$ if and only if $q>1$. As this condition is verified by $q^{+}$only, the value function and the optimal control are given by (13) and (12), respectively.

Furthermore, it can be proved that the hypotheses of Theorem A.1 are satisfied.

(ii) When $0<x<u$ and $k<r$, then $0<q^{-}<1<q^{+}$. The function $G_{u}$ must be a strictly concave and increasing solution of (32), with $G_{u}(u)=1$ and $\boldsymbol{\Lambda}_{G_{u}}$ can be obtained from (31). Now, $G_{u}(x)=p x^{q}, 0<x<u$, is simultaneously increasing and concave if and only if $0<q<1$. It is verified by $q^{-}$only, so the value function and the optimal control are given by (16) and (15), respectively. The remaining hypotheses of Theorem A.1 are easily verified.

Proof of Proposition 4.2. Theorem A.1 is valid for minimization if we change sup by inf in the corresponding HJB equation and if the solution $V$ is strictly convex and decreasing. Suppose that $0<x<u$. It is easy to check that $V_{u}$ as given in (22) is a strictly convex and decreasing solution of

$$
(r-k) x V^{\prime}(x)-\frac{1}{2} \theta^{\top} \theta \frac{\left(V^{\prime}(x)\right)^{2}}{V^{\prime \prime}(x)}+1=0, \quad 0<x<u,
$$

with $V_{u}(u)=0$. By (31), $\boldsymbol{\Lambda}_{V_{u}}$ is the vector appearing in (21). Furthermore, it can be proved that the hypotheses of Theorem A.1 are satisfied.

Proof of Proposition 5.1. It is immediate to check that $W$ is a solution of the HJB equation (23) in each case (note that, in the underfunded case, the aim is to minimize instead of maximize; hence in this region sup must be changed by inf in the HJB equation (23)). Furthermore, $W$ is strictly concave (convex) in the overfunded (underfunded) region. It remains to prove the transversality condition

$$
\lim _{t \rightarrow \infty} \mathbb{E}_{x} \mathrm{e}^{-\rho t} W\left(X^{\boldsymbol{\Lambda}_{W}}(t)\right)=0
$$

(see Fleming and Soner (1993, Theorem 9.1)).

(i) Eq. (9) is now

$$
\mathrm{d} X^{\boldsymbol{\Lambda}_{W}}(t)=\left(r-k-\frac{1}{\gamma-1} \theta^{\top} \theta\right) X^{\boldsymbol{\Lambda}_{W}}(t) \mathrm{d} t-\frac{1}{\gamma-1} \theta^{\top} X^{\boldsymbol{\Lambda}_{W}}(t) \mathrm{d} w(t) .
$$

Applying Itô's Lemma to $f(t)=\mathrm{e}^{-\rho t} W\left(X^{\boldsymbol{\Lambda}_{W}}(t)\right)$, we find

$$
\mathrm{d} f(t)=\left(-\rho+(r-k) \gamma-\frac{\theta^{\top} \theta}{2} \frac{\gamma}{\gamma-1}\right) f(t) \mathrm{d} t-\left(\frac{\gamma}{\gamma-1} \theta^{\top}\right) f(t) \mathrm{d} w(t) .
$$

Taking expectations, and because the stochastic integral $\int_{0}^{t} f(s) \mathrm{d} w(s)$ is a martingale, we have

$$
\begin{aligned}
\mathbb{E}_{x} \mathrm{e}^{-\rho t} W\left(X^{\Lambda_{W}}(t)\right) & =\mathbb{E}_{x} f(t) \\
& =\frac{\xi}{\gamma}|x|^{\gamma} \mathrm{e}^{-\left(\rho-(r-k) \gamma+\left(\theta^{\top} \theta \gamma\right) /(2(\gamma-1))\right) t} \\
& =\frac{\xi}{\gamma}|x|^{\gamma} \mathrm{e}^{-t / \xi} \rightarrow 0 \quad \text { as } t \rightarrow \infty,
\end{aligned}
$$


provided $\xi$ as given in (24) is positive.

(ii) It is completely similar to the case above. Now $\mathbb{E}_{x} \ln X^{\boldsymbol{\Lambda}_{W}}(t)=\ln x+\left(r-k+\frac{\theta^{\top} \theta}{2}\right) t$, since $Y=\ln X^{\boldsymbol{\Lambda}_{W}}$ satisfies $Y(t)=\ln x+\left(r-k+\frac{\theta^{\top} \theta}{2}\right) t+\theta^{\top} w(t)$, hence the transversality condition (34) is fulfilled.

\section{References}

Boulier, J.F., Trussant, E., Florens, D., 1995. A dynamic model for pension fund management. In: Proceedings of the 5th AFIR International Colloquium 1, pp. 361-384.

Bowers, N.L., Hickman, J.C., Nesbitt, C.J., 1979. The dynamics of pension funding: Contribution theory. Transactions of the Society of Actuaries 31, 93-119.

Browne, S., 1997. Survival and growth with a fixed liability: Optimal portfolios in continuous time. Mathematics of Operations Research 22, 468-493.

Browne, S., 1998. The return on investment from proportional portfolio strategies. Advances in Applied Probability 30, $216-238$.

Browne, S., 1999. Beating a moving target: Optimal portfolio strategies for outperforming a stochastic benchmark. Finance and Stochastics 3, 275-294.

Cairns, A.J.G., 2000. Some notes on the dynamics and optimal control of stochastic pension fund models in continuous time. Astin Bulletin 30, 19-55.

Cairns, A.J.G., Parker, G., 1997. Stochastic pension fund modelling. Insurance: Mathematics and Economics 21, 43-79.

Fleming, W.H., Soner, H.M., 1993. Controlled Markov Processes and Viscosity Solutions. Springer Verlag, New York.

Haberman, S., Butt, Z., Megaloudi, C., 2000. Contribution and solvency risk in a defined benefit pension scheme. Insurance: Mathematics and Economics 27, 237-259.

Haberman, S., Sung, J.H., 1994. Dynamics approaches to pension funding. Insurance: Mathematics and Economics 15, $151-162$.

Harrison, J.M., 1985. Brownian Motion and Stochastic Flow Systems. Wiley \& Sons, New York.

Karlin, S., Taylor, H.M., 1981. A Second Course on Stochastic Processes. Academic Press, New York.

Krylov, N.V., 1980. Controlled Diffusion Processes. Springer, New York.

Josa-Fombellida, R., Rincón-Zapatero, J.P., 2001. Minimization of risks in pension funding by means of contribution and portfolio selection. Insurance: Mathematics and Economics 29, 35-45.

Josa-Fombellida, R., Rincón-Zapatero, J.P., 2004. Optimal risk management in defined benefit stochastic pension funds. Insurance: Mathematics and Economics 34, 489-503.

Merton, R.C., 1971. Optimal consumption and portfolio rules in a continuous-time model. Journal of Economic Theory 3, $373-413$.

O'Brien, T., 1987. A two parameter family of pension contribution functions and stochastic optimization. Insurance: Mathematics and Economics 6, 129-134.

Pestien, V.C., Sudderth, W.D., 1985. Continuous-time red and black: How to control a diffusion to a goal. Mathematics of Operations Research 10, 599-611.

Siegmann, A.H., Lucas, A., 1999. Continuous-time dynamic programming for ALM with risk-averse loss functions. In: Proceedings of the 9th AFIR International Colloquium 2, pp. 183-193. 